\title{
Molnupiravir Extends COVID-19 Viral Phase, Evidenced by the High Frequency of Rare and Dangerous Mutations in SARS-COV-2
}

\section{Abstract}

This paper analyzes SARS-COV-2 mutations data from Merck's Molnupiravir trials, in the larger context.

- 5-day treatment with Molnupiravir caused the appearance and selection (to a frequency $>5 \%$ ) of two of the most dangerous spike mutations - E484K and $\mathrm{P} 681 \mathrm{H}-$ in multiple patients of a very small group (2/202 and 4/202, respectively).

- Molnupiravir disproportionately increases the frequency of dangerous and unusual mutations

- Molnupiravir worsens COVID-19 in patients, especially those who start treatment within 3 days of symptom onset. Some theoretically possible mechanisms causing this include acute bone marrow disorder and/or the generation of immune-evasive or even immunosuppressive viral genomes.

- These mechanisms are likely to extend the virus shedding period in a substantial number of patients. The virus shed by these patients would be highly mutated and likely selected toward virulence.

- Molnupiravir allows for virus diversification in the treated minority and purification in the untreated, a luxury rarely experienced by any virus in the nature.

- Merck failed to collect enough data about Molnupiravir driven mutations.

- For each important safety event, the collected data represents a few realizations of a random variable with unknown heavy tailed statistical distribution. Merck incorrectly treated this data as worst-case scenarios.

\section{Introduction}

Molnupiravir (also known as EIDD-2801 and MK-4482 and a variety of trade names, including Lagevrio "MOLN") converts into its active metabolite NHC (EIDD-1931), within the body. MOLN's mechanism of action is introducing large number of mutations in the SARS-COV-2 genome. Its wide-spread use is creating a global catastrophic threat ${ }^{1}$ by diversifying the SARS-COV-2 genome and breeding more dangerous variants.

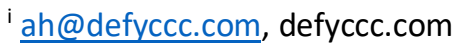


The main data sources (Merck Briefing ${ }^{2}$ and FDA Backgrounder ${ }^{3}$ ) have been downloaded from the FDA AMDAC meeting page ${ }^{4}$.

Bangladesh $^{5}$, the Philippines ${ }^{6}$, and Vietnam ${ }^{7}$ started to use MOLN widely, possibly under the incorrect impression that the UK gave it final authorization. Philippines' president even personally promoted it. France has properly rejected MOLN ${ }^{8}$.

\section{Data}

The mutations data comes from the Merck Backgrounder ${ }^{2}$ pp.34-35 and FDA Briefing ${ }^{3}$ pp. 32-36. The FDA received all its data from Merck but ran additional analysis on mutations in the virus' spike gene. The data from the trial MK-4482-001 (P001, Part 1, hospitalized patients) (ref. ${ }^{3}$, Table 9) is for 89 MOLN patients. The data from MK-4482-002 (P002, Part 1, outpatients and different dosage) (ref. ${ }^{3}$, Table $^{8}$ ) is for 113 MOLN patients. Each table contains pooled data, including 200 mg, 400 mg, and $800 \mathrm{mg}$ doses. Merck Backgrounder ${ }^{2}$ also contains data for mutations in the NSP12-14 regions. The available information includes only mutations outside in the S-protein and ORF1b genes .

Merk ${ }^{2}$ states (p. 34): "The analysis focused on treatment-emergent variants that occurred at a frequency of $\geq 5 \%$ in $\geq 2$ participants in the combined MOV groups."

Smaller numbers of placebo patients were used for comparison. Because of the similarity of the results, they are combined here.

The following Table 1 shows mutations that were mentioned in either document and achieved $>5 \%$ frequency in at least two patients. Only MOLN patients had mutations matching this criterion.

Table 1. Mutations in at least two MOLN patients with frequency $>5 \%$

\begin{tabular}{|l|l|l|}
\hline Mutation & Patients & Remarks \\
\hline S:del141/144 & 3 & $\begin{array}{l}\text { Additional overlapping } \\
\text { mutations }\end{array}$ \\
\hline S:del145 & 3 & $\begin{array}{l}\text { Additional overlapping } \\
\text { mutations }\end{array}$ \\
\hline S:G261I/V & $2(1+1)$ & \\
\hline S:T385I & 2 & Mutation of Concern \\
\hline S:E484K & 2 & Mutation of Interest \\
\hline S:P681H & 4 & \\
\hline S:A1022T & 2 & \\
\hline nsp12:A446T & 2 & \\
\hline nsp14:A220S/V & $2(1+1)$ & \\
\hline nsp14:V466I & 2 & \\
\hline
\end{tabular}

The data represents samples after 5 days of the treatment. The mutations load in the MOLN group is many times higher than in the placebo group. A suggested cause of deletions was errors in the repair, in proofreading (FDA Briefing 3 p. 35).

Methods and Results

Frequency of Mutations under MOLN

The trial patients were given MOLN doses between $200 \mathrm{mg}$ and $800 \mathrm{mg}$ (majority), twice a day for 4-5 days, starting after symptoms onset. Symptoms onset is typically close to the maximum viral load (ref. ${ }^{9}$, 
Fig 1), although there is some individual variation. Non-hospitalized patients started receiving MOLN within 5 days from symptoms onset; hospitalized patients started later. In the normal cause of disease, the viral load remains constant or decreases at that time. If MOLN does not extend the viral period, the virus would only have 1-5 generations (of 24 hours each) to mutate under the influence of MOLN. It would then have to undergo selection, in order to reach the $5 \%$ frequency, measured by PCR tests. This is impossible under normal circumstances. The only explanation for the fact that this happened in $35 \%$ of the sequenced MOLN patients $\left(\mathrm{FDA}^{3}\right)$ is that MOLN slows down the process of virus clearing. This can happen if MOLN poisons the bone marrow (Merck ${ }^{2} \mathrm{pp}$. 15-16) quickly, or disproportionately creates immunity evading mutations. This conclusion is consistent with the results of MK-4482-002 Phase 3 Part 2 trial, in which MOLN patients had a higher risk of hospitalization and/or death than the placebo patients ${ }^{10}$. Part 1 of that trial is less relevant because it was conducted or reported incorrectly ${ }^{10}$. But in both Part 1 and Part 2 of the trial, the patients who started MOLN within 3 days of symptoms onset had significantly worse outcomes compared with those who started later. This is consistent with pro-viral, rather than anti-viral activity of MOLN.

\section{Non-MOLN Mutations}

Some spike mutations (del145, A262S, S297L, and S:S884F) had frequencies crossing the $5 \%$ line in one placebo patient each. That might be explained by their presence from before intervention at frequencies $<5 \%$. Each of these mutations has at least a moderate presence in GISAID. Also, each of the mutations was also present in one or more MOLN patients, raising a theoretical possibility of infection of placebo patients by MOLN patients.

\section{Dangerous Mutations}

In the 202 patients treated with MOLN, and having virus sequenced before and after the treatment, the drug was able to mutate the coronavirus to get $\mathrm{S}: \mathrm{E} 484 \mathrm{~K}$, the most dangerous spike mutation (the mutation of concern ${ }^{11}$ ), and $\mathrm{S}: \mathrm{P} 681 \mathrm{H}$, which is somewhere between $\# 2$ and \#6 of the top mutations of interest ${ }^{11}$. Each of these mutations was present with a frequency $>5 \%$ in $2-4$ MOLN patients!

Yes, these mutations have also happened in nature, but it took many months and tens millions of hosts to get variants containing them in such high quantities. E484K and $\mathrm{P} 681 \mathrm{H}$ achieved a $5 \%$ prevalence in GISAID in February 2021 and November 2020, respectively. MOLN created and ensured the selection of these mutations in a group of two hundred hosts within five days!

E484K breaks many epitopes for neutralizing antibodies. Just like MOLN enabled the coronavirus to "find" this mutation, it is likely to find other antibodies-evading mutations in a small percent of patients. $\mathrm{P} 681 \mathrm{H}$ eases cleaving and entrance of the coronavirus into the cell. These mutations are likely to prolong the shedding period and thus be transferred to others.

\section{Rare mutations}

The rare substitutions in MOLN patients are even more worrisome. Normally, for SARS-COV-2 in humans, the most frequent substitution is transition $\mathrm{C}>\mathrm{U}^{\mathrm{ii}}$ at $\% 48 \%$. The least frequent transition is $\mathrm{G}>\mathrm{A}$ at $5 \%$ of all ${ }^{12}$. The $\mathrm{G}>\mathrm{U}$ transversion is a more frequent one, at $13 \%$.

\footnotetext{
ii All T's are replaced by $\mathbf{U}$ 's for uniformity. $\mathbf{U}$ is equivalent of $\mathbf{T}$ in the designation of nucleotides. This paper is intended for various audiences.
} 
The following rare mutations were found in MOLN trial patients (at least in two at the frequency $>5 \%$ ):

Mutation S:G261I does not appear among >5.5M sequences in GISAID, as interpreted by Outbreak.info ${ }^{11}$. This mutation cannot be accomplished by a single nucleotide mutation. It requires a double-nucleotide mutation $G G>A U^{13}$. But it happened, under the influence of MOLN, in 2 of the 202 patients, and it was selected to $>5 \%$. This was ignored.

Mutation S:G261V does not appear in GISAID either. It only happens by G>U transversion. It is not rare. If it had a neutral fitness impact, it would be expected to appear in GISAID 100-200 times. This means that this mutation is usually very detrimental for the virus. Its unusual success under MOLN might be explained only if it appeared as part of a large synergistic mutations set.

Mutation nsp14:V466I does not appear in GISAID either. It only happens by G>A transition. If it had a neutral fitness impact, it would be expected to appear in GISAID 50 times. MOLN increases the frequency of $\mathrm{G}>\mathrm{A}$ transitions by $12 \mathrm{x}$, but even so, this mutation's success under MOLN would only be explained if it appeared as part of a large synergistic mutations set.

The propensity to create many synergistic mutations sets, containing mutations not recorded in nature, is another dangerous property of MOLN.

Mutation S:A1022T appears in GISAID seven times, which is less than expected. Everything said about nsp14:V466I applies to S:A1022T, to a lesser degree.

\section{7 mutations in one patient}

Merck ${ }^{2}$ statement: "One of these MOV participants had multiple spike changes ( $\triangle Y 145, N 501 Y$ and $\mathrm{P} 681 \mathrm{H})$ with a high number changes emergent at other genome locations. This participant was subsequently found to have switched genotypes between baseline (Clade 20E) and Day 3 (Clade 20B), suggesting either possible co-infection or sample processing error."

Clades $20 \mathrm{E}$ and 20B are rare, so co-infection is unlikely. A sample processing error is possible, but the researchers should have kept samples and to repeat the processing, or they could have taken another sample from the same patient. There were seven mutations in one patient. This result should not be dismissed. The variation in MOLN concentration over the time, the unknown interactions between MOLN and the host editing mechanism, and the possible suppression of the immune system by MOLN must all be carefully considered.

\section{Fallacies in the Official Analysis}

Merck's Self Contradictions

Merck's own data contradicts their claims presented in the slides for AMDAC ${ }^{14}$. For example:

Slide 16: "Errors observed with molnupiravir treatment were randomly distributed. No evidence of an increased rate of transition errors at any specific position or gene including the replicase or spike"

The appearance of mutations in the same codons and their rise above the $5 \%$ threshold, in multiple patients, is nowhere close to random distribution.

Logical Fallacies by Merck and the FDA

Merck and the FDA demonstrate other fallacies in analyzing the results. 
From Merck's briefing document ${ }^{2}$ :

"All of the emergent changes in spike in MOV participants were deletions or transversion errors; these types of changes are not associated with the MOA of MOV which results in transition errors (eg, $C$ to $U, G$ to A)." (p.34) MOA stands for "mechanism of action"

"the $P 681 \mathrm{H}$ substitution and $139-145$ region deletions were not transition errors and therefore, unlikely attributable to treatment with MOV." (p.34)

If results of trials contradict the theory, the theory is wrong. Merck claims other way around. It is wrong.

\section{Statistical Fallacies by Merck and the FDA}

Dismissing the appearance and near-fixation of various mutations, from extremely rare to extremely dangerous ones, cannot be justified. Outpatients who started MOLN treatment within 3 days from the symptom onset had $80 \%$ higher odds of hospitalization (Part 2$)^{10}$. In the MOLN treatment group, the relative growth of genomes carrying these dangerous mutations likely happened toward the end of the viral phase of the disease. However, other people would be infected by the virus with these mutations and incubate it from the beginning.

This Merck/FDA analysis also contains a statistical fallacy. The mutations data represents a few realizations of random variables with unknown probability distributions.

One random variable is the outcome of the patient (from $0=$ no harm to infinity=death). Another random variable is the outcome of the civilization, (from $0=$ no harm to infinity=globally devastating variants). Each of these distributions has an unknown mean and variance. The estimation is done from a tiny sample, having about a dozen events. The sampling results have heavy-tailed probability distributions. This means that the risk of the right tail events (individual death and civilization devastation) is not small.

\section{Discussion}

Unnatural Selection for Virulence

MOLN creates within-host diversity, which is then subjected to within-host natural selection. This selection is for higher virulence. Viral genomes that evade the human immune response, replicate faster and in higher amounts, and thus, survive and propagate. Genomes that suppress human immunity also get a fitness plus, because they extend the period in which they can hypermutate.

\section{Unnatural Uneven Increase in the Frequencies of Mutation Types}

MOLN not only increases the frequency of mutations, but it also changes the ratio between mutations. Merck's data shows that the frequency of $G>A$ transitions is increased by $12 x$, while the frequency of $C>$ $U$ transitions is increased only by $2 x$ (as measured, i.e., after intra-host selection; here, the rate or frequency of such mutations that survived intra-host selection is referred to as the mutation rate or frequency, respectively).

This sharply increases the danger of antigenic shifts toward more fit coronavirus variants, which are likely to be more virulent than the current ones. Ref. ${ }^{15}$ studied the phenomenon of increased discovery of functional proteins with the increase of mutation rates, and explained it as overcoming the "uniqueness sink". 
Each coronavirus genome can be represented as a vector in the space of genomes. This space has about 30,000 dimensions (the length of the genome), and the distance on it is measured by the number of nucleotide differences. The original Wuhan strain is taken as 0 . Each major SARS-COV-2 variant, from Alpha to Delta to Lambda, is near the local fitness maximum. Normally, the lineage explores its vicinity by small steps, corresponding to a relatively low number of mutations per copy. From the beginning of the pandemic, virus RNA replicated globally $\sim 10^{18}$ times. Most of these replications were in the Delta variant(s). A more fit genome is more likely to survive and propagate. The vicinity of the local optimum is well explored; each combination of small number of mutations from it was tried many times and found not to be a higher local maximum than few others (B.1.617.2, AY.4, AY4.2, AY.103 and so on). But it is not explored uniformly. Because $C>U$ transitions has an $\sim 10 x$ higher frequency than $G>A$, mutations sets that include $\mathrm{C}>\mathrm{U}$ but not $\mathrm{G}>\mathrm{A}$, at the first two positions in the codon, happened much more frequently than those containing $\mathrm{G}>\mathrm{A}$. Replications with two or more $\mathrm{G}>\mathrm{A}$ happened rarely. But as far as this frequency ratio does not change, each new replication in a major or old lineage is likely to create a genome that had already appeared many times. If it had high fitness, it would have led to another major variant (for as long as the fitness function does not change).

MOLN however, increases the frequency of $G>A$ transitions by $12 x$, and $A>G$ by $4 x$ (ref. ${ }^{16}$, Annex). Thus, the frequency of two $\mathrm{G}>\mathrm{A}$ transitions in one replication is increased by $12^{2}$, or $144 \mathrm{x}$, and so on. A large fraction of new mutation sets falls on unexplored territory. Some of these sets are synergistic, having a higher fitness than close local maxima. After discovering this higher ground, the virus continues exploring the genomes space from it, and finds a new local maximum. For us, this becomes a new variant.

\section{Unnatural and Pro-Viral Switching between High and Low Mutation Rates}

Some viruses have high mutation rates, some have low ones. The higher mutation rates increase the speed of the virus' adaptation, at the expense of a higher fraction of unfit virions. MOLN allows SARSCOV-2 to switch between these and benefit from both a high and a low mutation rate. The high mutation rate takes place in the patients treated with MOLN. That highly mutated virus is then transmitted to other people, who are not treated with MOLN. In these "untreated" patients, the different versions of the virus recombine and undergo selection at low mutation rates, finding local maxima.

In computer science, the optimization process that MOLN allows for SARS-COV-2 is called simulated annealing ${ }^{17}$. Notice that the entropy (RNA errors load) decreases gradually, as the transmitted mutated virus recombines in the new hosts, and stronger lineages undergo purification.

\section{Conclusion}

MOLN's action on SARS-COV-2 in humans is very different from its action in-vitro and in mice and rats. Humans have much more elaborate in-cell anti-viral host editing mechanisms ${ }^{18}$. MOLN experiments on humans have failed, and it is time to stop them.

\section{No Competing Interests}

The author declares no competing interest. No funding was provided for this work.

\section{Reference}

The most important references are ${ }^{2}$ and ${ }^{3}$. 
1. Anonymous A. Treating a Pandemic Respiratory Disease with a Mutagen Is a Doomsday Scenario. Preprints; 2021. doi:10.22541/au.163854323.34557301/v1

2. Merck Backgrounder. Molnupiravir Oral Treatment of COVID-19, Advisory Committee Briefing Materials. Published online November 26, 2021. https://www.fda.gov/media/154421/download

3. FDA CDER Briefing. FDA Briefing Document. Published online November 26, 2021. https://www.fda.gov/media/154418/download

4. AMDAC. November 30, 2021: Antimicrobial Drugs Advisory Committee Meeting Announcement 11/30/2021 - 11/30/2021. FDA. Published November 30, 2021. https://www.fda.gov/advisorycommittees/advisory-committee-calendar/november-30-2021-antimicrobial-drugs-advisorycommittee-meeting-announcement-11302021-11302021

5. Correspondent S, bdnews24.com. Bangladesh makes Merck's COVID-19 pill molnupiravir available. Published December 9, 2021. https://bdnews24.com/health/2021/11/09/bangladesh-makesmercks-covid-19-pill-molnupiravir-available

6. Lalu GP. Molnupiravir available to OVP telemedicine patients under prescription. INQUIRER.net. Published November 23, 2021. Accessed December 4, 2021.

https://newsinfo.inquirer.net/1518590/molnupiravir-available-to-ovps-telemedicine-patients-whoare-prescribed-to-take-it

7. DTI News. Vietnam expands Molnupiravir usage for home Covid-19 treatment | DTiNews - Dan Tri International, the news gateway of Vietnam. Published December 13, 2021. http://dtinews.vn/en/news/017002/77085/vietnam-expands-molnupiravir-usage-for-home-covid19-treatment.html

8. Endpoints News. Covid-19 roundup: French regulators not ready to give molnupiravir the OK; Brii claims China's first antibody is effective against Omicron. Endpoints News. Published December 13, 2021. https://endpts.com/covid-19-roundup-french-regulators-not-ready-to-give-molnupiravir-theok-chinas-first-antibody-cocktail-proves-effective-against-omicron/

9. FLCCC Alliance. FLCCC Protocols - A Guide to the Management of COVID-19. Published online November 12, 2021. https://covid19criticalcare.com/wp-content/uploads/2020/12/FLCCCProtocols-\%E2\%80\%93-A-Guide-to-the-Management-of-COVID-19.pdf

10. Goldstein L. Re-analysis of Molnupiravir Trials, Phase II/III. Tria/SiteNews. Published online November 28, 2021. https://trialsitenews.com/re-analysis-of-molnupiravir-trials-phase-ii-iii/

11. Outbreak.info. Outbreak.Info.; 2021. https://outbreak.info

12. Tonkin-Hill G, Martincorena I, Amato R, et al. Patterns of within-host genetic diversity in SARS-CoV2. Neher RA, Sawyer SL, Neher RA, Lauring AS, eds. eLife. 2021;10:e66857. doi:10.7554/eLife.66857

13. GenBank. Severe acute respiratory syndrome coronavirus 2 isolate Wuhan-Hu-1, complete genome. Published online July 18, 2020. http://www.ncbi.nlm.nih.gov/nuccore/NC 045512.2 
14. Merck. Molnupiravir. Presented at: November 30, 2021.

https://www.fda.gov/media/154472/download

15. Drummond A, et al. Why High-error-rate Random Mutagenesis Libraries are Enriched in Functional and Improved Proteins - ScienceDirect. Published online 2005. Accessed November 11, 2021. https://www.sciencedirect.com/science/article/abs/pii/S0022283605005541

16. Goldstein L. Mildness of Host-Induced Mutations in SARS-COV-2. Preprints; 2021. doi:10.22541/au.163898733.32583439/v1

17. Microsoft. Simulated annealing - Azure Quantum. Published 2021. https://docs.microsoft.com/enus/azure/quantum/optimization-simulated-annealing

18. Stavrou S, Ross SR. APOBEC3 Proteins in Viral Immunity. J Immunol. 2015;195(10):4565-4570. doi:10.4049/jimmunol.1501504 